Elise Klein, Stefanie Jung, and Liane Kaufmann

\title{
A neuropsychological perspective on the development of and the interrelation between numerical and language processing
}

\section{Introduction}

Impairments in number processing (e.g., dyscalculia, acalculia) are often associated with impairments in language processing (e.g., dyslexia, aphasia) in both developmental disorders and adult neurological syndromes (e.g., Geary, 1993; Willmes, 2008). However, number processing deficits in aphasics are not necessarily reducible to the language impairment (Basso et al., 2005). Recent studies in mathematical experts even suggest the existence of two distinct, non-overlapping networks for mathematics and language (Amalric \& Dehaene, 2018). This underscores the importance to understand the neuropsychological foundations of mathematics and language processing. The main aim of this chapter is to present the current neuropsychological literature of numerical cognition so that in a second step similarities and intersections with language processing can be briefly outlined.

\section{Number processing and mental arithmetic}

\subsection{Models for numerical cognition}

In the past four decades, various theoretical models have been proposed to provide a conceptual framework for the cognitive components involved in number processing and mental arithmetic (e.g., Campbell, 1994; Cipolotti \& Butterworth, 1995; Dehaene, 1992; McCloskey, 1992; for an overview, see Deloche \& Willmes, 2000). These models differ in the number and type of postulated representations and their interactions. The goal of all attempts was to develop a sufficiently detailed model to explain the numerical and arithmetical skills of adults with and without specific (learning) impairments. In the last two decades, the Triple-Code Model (TCM) of Dehaene and its elaborations (1992; Dehaene \& Cohen, 1995, 1997; Dehaene et al., 2003) became the most influential model in numerical cognition because of its unique integration of behavioral and neurofunctional

○ Open Access. () 2021 Elise Klein et al., published by De Gruyter. (c))BY-NC-ND This work is licensed under the Creative Commons Attribution-NonCommercial-NoDerivatives 4.0 International License. 
aspects. However, it is important to note that the original TCM is based on adults (i.e., mature brain systems) only (Kaufmann et al., 2013).

\subsection{The Triple-Code Model}

One of the most important postulates of the TCM (Dehaene, 1992) is the distinction between a number magnitude representation on the one side and arithmetic fact retrieval from memory on the other side. Moreover, a visual number form representation in bilateral fusiform areas is assumed for recognizing Arabic digits. Fig. 1.

As regards the number magnitude representation, a bilateral fronto-parietal network around the intraparietal sulcus (IPS) subserves the representation and mental manipulation of numerical quantities (e.g., $28+52$ ). In contrast, simpler tasks such as multiplication with one-digit numbers (e.g., $3 \times 2$ ) are solved by arithmetic fact retrieval in a left-hemispheric network including perisylvian language areas and the angular gyrus (Dehaene et al., 2003).

It is important to note that these representations can dissociate. For instance, patients suffering from a left-hemispheric stroke can present with a selective deficit of rote verbal knowledge (including multiplication facts, e.g., Zaunmuller et al., 2009) with preserved semantic knowledge of numerical quantities. On the other hand, patients with IPS lesions can show specific impairments of quantitative numerical knowledge (e.g., in subtraction), while knowledge of rote arithmetic facts is preserved (e.g., in simple multiplication; Dehaene \& Cohen, 1997). These double dissociations suggest that numerical knowledge is processed in different codes within distinct cerebral areas.

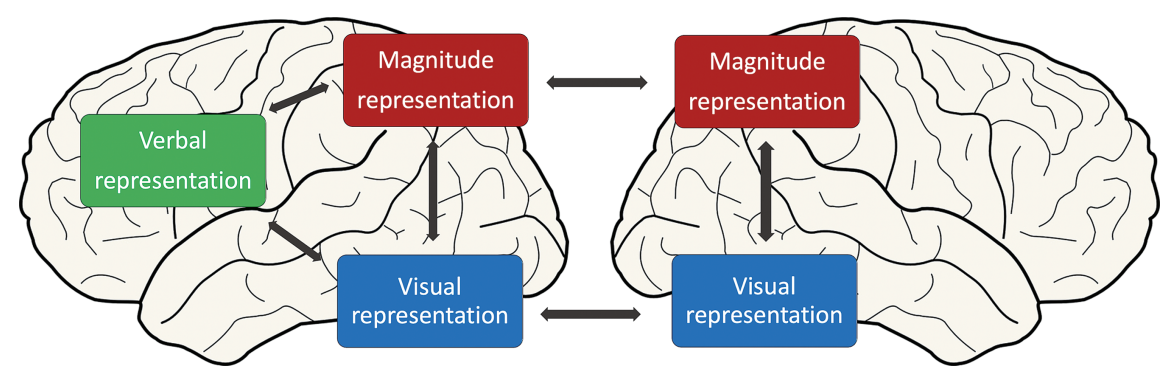

Fig. 1: Schematic Integration of Functional and Anatomical Assumptions of the Triple-Code Model (modified from Dehaene \& Cohen, 1995). The brain regions postulated for the three codes (magnitude representation, verbal representation, visual representation) are projected onto lateral views of the left and right hemispheres. Arrows represent theory-based assumptions about transcoding pathways between codes rather than empirically substantiated white matter connections. 


\subsubsection{Neurofunctional correlates of number magnitude}

The TCM incorporates evidence from studies on brain-lesioned patients, human functional neuroimaging, and primate neurophysiology in indicating that numerical cognition is subserved by a fronto-parietal network centered around the IPS. In particular, the IPS is dedicated to the mental manipulation of numerical quantities, if, for instance, it has to be decided which of two numbers is the numerically larger one. Here, numbers are coded as analogue magnitudes in an abstract notation-independent format (Piazza et al., 2007; but see Cohen Kadosh et al., 2007), each of them activating a small segment on a nonverbal, logarithmically compressed left-to-right oriented quantitative representation called "mental number line.” The bilateral IPS are connected through transcallosal fibers, which enable the interplay between both hemispheres when semantic number magnitude is processed (Ratinckx et al., 2006). The IPS is active even in numerical tasks that do not necessarily require quantity processing (Eger et al., 2003, Klein et al., 2010) or that present numerical stimuli unconsciously (Naccache \& Dehaene, 2001). In more complex numerical problems, the quantity-specific IPS is complemented by (pre)frontal areas involved in more general cognitive processes such as attention, working memory, or problem solving. However, the involvement of prefrontal cortices was only vaguely specified in the TCM. Based on an fMRI meta-analysis, Arsalidou and Taylor (2011) suggested a modification and extension of Dehaene's model specifying a refined picture of (pre)frontal functions and, thus, of supporting and domain-general functions implicated in solving arithmetic tasks.

Finally, the TCM considers the bilateral posterior superior parietal lobe (PSPL) to support magnitude processing via mental orientation of attention on the mental number line (Dehaene et al., 2003).

\subsubsection{Neurofunctional correlates for arithmetic fact retrieval}

Neurofunctional evidence on arithmetic fact retrieval primarily comes from studies that pursued the acquisition of arithmetic facts by means of drill training of difficult multiplication problems (e.g., $43 \times 9=\ldots$ _; Delazer et al., 2003). A consistent finding was a switch from magnitude-related processing to verbally-mediated arithmetic fact retrieval with learning, reflected by a shift in activation from the bilateral fronto-parietal network of number processing to a left-hemispheric network including perisylvian language areas and angular gyrus (AG). The authors interpreted this to reflect a shift from quantity-based and working memory demanding computations to automatic retrieval of arithmetic 
facts from long-term memory. Consequently, it was argued that the left AG constitutes a key area for these retrieval processes (e.g., Dehaene et al., 2003).

In recent years mounting evidence suggested the additional involvement of long-term memory processes, subserved by the medial temporal lobe centered around the left hippocampus (Klein et al., 2016; Menon, 2016), while the role of the AG for arithmetic fact retrieval was challenged (e.g., Bloechle et al., 2016). The involvement of long-term memory areas in fact learning was corroborated by diffusion tensor imaging (DTI) data showing a significant increase of structural connectivity in fibers encompassing the left hippocampus but not the left AG following drill training of multiplication facts (Klein et al., 2019). Converging evidence for a central role of the hippocampus in mental arithmetic in general and arithmetic fact retrieval in particular comes from developmental research on functional connectivity in arithmetic fact learning (e.g., Rosenberg-Lee et al., 2018). For instance, hippocampal activation and functional connectivity increased with the acquisition of mathematical knowledge following a short-term training. However, the question whether the role of the hippocampus in fact learning is time-limited is not resolved yet. Recent neuropsychological single-case studies suggest that the hippocampus might be necessary only for the consolidation of arithmetic facts in memory rather than the retrieval of well-consolidated facts from memory (Delazer et al., 2019).

Taken together, it is assumed that the magnitude representation is supported by both cerebral hemispheres, while the verbal representation of arithmetic facts is situated in the left hemisphere only. Thus, only a bilateral parietal lesion would lead to a permanent impairment of the quantitative number representation.

\subsection{Developmental models}

Compared with the adult literature, there are far fewer studies investigating the neurofunctional correlates of number processing and calculation in children. A quantitative meta-analysis of these fMRI studies in children aged 14 years or younger is provided by Arsalidou et al. (2018). Interestingly, beyond brain areas reported to be associated with symbolic and non-symbolic mental arithmetic in adults (Arsalidou \& Taylor, 2011), the authors identified additional brain areas (i.e., the insula and the claustrum) supporting number processing in children (Arsalidou \& Taylor, 2018). These brain regions are not typically considered in numerical cognition models in adults.

Notably, neurofunctional trajectories of typically and atypically developing children may manifest differently at both the behavioral and the brain levels. 
Below, we will focus on specific factors known to influence children's numerical development, namely, age, math proficiency, and notation.

\subsubsection{Age effects}

There is accumulating evidence that cerebral activation patterns in response to number processing are clearly age dependent (see the meta-analysis of developmental fMRI studies, Ashkenazi et al., 2013; Kaufmann et al., 2011). Compared with adults, children were found to activate more anterior (intra)parietal regions upon solving number magnitude comparison tasks (despite comparable accuracies and reaction times). A plausible explanation for the more anterior parietal activations (neighboring the postcentral gyrus, which is known to host sensory functions of the hand and fingers) is that children have a stronger need to rely on finger-based solution strategies (Kaufmann et al., 2011). Furthermore, when compared to adults, children are found to stronger recruit (pre)frontal brain regions that have been interpreted to reflect more effortful processing. Interestingly, upon investigating number processing in prematurely born six- and seven-year-old children that were just about to start formal education, Klein et al. (2018) found that gestational age predicted the frontal-to-parietal activation shift associated with number magnitude processing.

\subsubsection{Effects of math proficiency}

It is important to note that beyond the age of six or seven years (i.e., when formal schooling starts), age effects are inevitable confounded by math competency. Indeed, converging evidence suggests that with increasing age and schooling, cerebral activations associated with number processing become stronger in the posterior parietal cortex (including the IPS; symbolic number processing: Kaufmann et al., 2006; non-symbolic number processing: Ansari \& Dhital, 2006; Cantlon et al., 2006; addition and subtraction:, Rivera et al., 2005). Hence, math competency might be a modulating factor for the ease with which children access number representations (e.g., Bugden \& Ansari, 2011).

\subsubsection{Notation effects}

In children, different number notations are thought to be processed by distinct mental representations that become more overlapping with increasing age and 
math proficiency (Kucian \& Kaufmann, 2009). Contrary to the assumption of an abstract number magnitude representation (TCM, Dehaene, 1992), typically developing children were found to process non-symbolic and symbolic magnitudes in distinct parietal and extra-parietal brain regions (Kaufmann et al., 2011): non-symbolic number processing yielded predominantly right (intra)parietal activations, located more anteriorly than the bilateral (intra)parietal activations associated with children's symbolic number processing.

With respect to calculation tasks, 9- to 12-year-old typically developing children revealed distinct activation pattern for symbolic (i.e., Arabic digits and number words) and non-symbolic (i.e., dot arrays) number formats upon solving simple subtraction tasks (Peters et al., 2016). While subtraction with symbolic formats yielded parietal activations (i.e., AG and supramarginal gyri), subtraction with non-symbolic format led to parietal and extra-parietal activations (i.e., middle occipital and superior parietal lobes, superior frontal gyrus and insula). Most likely, these differential activation patterns reflect differences in strategy use.

\section{Neuropsychology of numerical cognition}

The scientific interest in numerical deficiencies is quite young. It was only at the beginning of the twentieth century that Henschen (1919) coined the term “acalculia” by systematically describing acquired numerical deficits in braindamaged patients. As regards innate numerical impairments in children, it took another 50 years before Kosc (1974) first introduced the term "dyscalculia." Notably, research on developmental dyscalculia and acquired acalculia has been separated from the beginning without systematic joint consideration and evaluation of the existing empirical evidence.

\subsection{Acquired acalculia}

Acquired acalculia designates the loss or impairment of the formerly intact ability to deal with numbers and/or to calculate following acquired brain pathology. In particular, up to $50 \%$ of patients with left-hemispheric and up to $30 \%$ of patients with right-hemispheric lesions suffer from acquired acalculia (Basso et al., 2005). Acalculia is often, but not necessarily, associated with other cognitive impairments such as language disturbances (Ardila \& Rosselli, 2002, for a review).

The symptomatology of acalculia is not homogeneous (see Ardila \& Rosselli, 2002; Domahs \& Delazer, 2005, for reviews). This led to clinically oriented 
classifications into subtypes being proposed already in the early stages of research on acalculia (e.g., however, see the single-route model proposed by McCloskey, 1992). Indeed, several case studies in the 1990s clearly showed that specific subcomponents of numerical cognition can be impaired differentially.

Generally, the symptomatology of acquired acalculia can be divided into (i) impairments of a (quantitative) magnitude representation and (ii) calculation impairments (oral and/or written), which can be further subdivided into (a) impairments of arithmetical fact retrieval and (b) impairments of procedural as well as conceptual arithmetic knowledge. Additionally, (iii) transcoding impairments are frequently observed in patients with left-hemispheric lesions. Importantly, these capacities can be affected independently (Domahs \& Delazer, 2005 for a review). This led Dehaene (1992) to formulate the multi-componential TCM, which assumes that numerical knowledge is processed in different formats within distinct cerebral areas.

\subsubsection{Impairments of the (quantitative) magnitude representation}

Problems with processing abstract numerical magnitudes have been observed less frequently than language-related errors. The postulated bilateral (i.e., redundant) representation of numerical quantity in the IPS makes it less vulnerable to focal brain damage. There are only a few patients reported in the literature suffering from unilateral IPS lesions of the language-dominant hemisphere who revealed problems in dealing with abstract quantities (Dehaene \& Cohen, 1997; Delazer \& Benke, 1997; Lemer et al., 2003). In these cases, counting or reciting multiplication tables from memory as well as reading aloud of numbers or transcoding of numerical symbols like dot patterns was preserved. In contrast, number magnitude comparison of Arabic digits was affected as well as number bisection (e.g., "What is the numerical middle between the two outer numbers 23 and 27?"). Simple subtraction tasks, which are not assumed to be solved via arithmetic fact retrieval from long-term memory, led to erroneous or no responses. Approximate calculation was impossible. Since number magnitude comparison of regularly placed sets of dots was impaired as well, the patients' problems with numerical magnitudes were not notation specific.

Delazer et al. (2006) reported a case of bilateral posterior cortical atrophy. The patient made errors in both a production and a verification version of the number bisection task (e.g., ls the middle number of this triplet also the numerical middle?” e.g., 23_26_29”; “23_25_29”). Similarly, approximate calculation tasks in which the less deviating alternative had to be selected quickly from two wrong solutions to an arithmetic problem were impaired. 
Single-case studies also show that numerical knowledge can dissociate from non-numerical knowledge at the level of semantic processing. For instance, Cipolotti et al. (1991) reported a patient who was completely dysgraphic and dyslexic for all kinds of material, but showed preserved oral performance for words except for numerals above four. In particular, the patient could not discriminate Arabic digits from meaningless shapes or numerals from nonwords, nor could she produce the direct "neighbors" of a number word presented auditorily or do numerical magnitude comparisons for number words above four.

\subsubsection{Impairments of arithmetical fact retrieval}

Impairments of arithmetic fact retrieval are seen much more frequently. They comprise errors in highly overlearned simple addition and subtraction problems with numbers below 20, and in simple multiplication for arithmetical tables up to $9 \times 9$, which are typically retrieved from declarative long-term memory in healthy adults. Erroneous responses to simple multiplication tasks in acalculia tend to be from the same multiplication table or a close entry from another table (i.e., within-table errors, McCloskey et al., 1985; e.g., “7 $\times 8=48$ ” or "7 $\times 8=54$,") and not from a more distant entry or even a non-table response. Problems with arithmetic fact retrieval are not necessarily related to higher error rates only; they can also manifest in substantially longer response times, indicating the use of calculation routines or strategies in case of hampered or impossible fact retrieval (Warrington, 1982).

Zaunmuller et al. (2009) reported a patient with a severe impairment of multiplication fact retrieval following a left-hemispheric lesion of the middle cerebral artery. After a customized arithmetic fact drill training over 30 days, the patient's multiplication performance improved significantly, accompanied by a shift of activation to the contralesional right AG.

However, some studies challenge the view that simple problems are always retrieved from memory (e.g., LeFevre et al., 1996). Also Domahs and Delazer (2005) pointed out that a precise definition of what constitutes a number fact is not available. Multiplications with zero or with 10 are considered to be rule-based and have been shown to dissociate from "proper" multiplication problems (Pesenti et al., 2000).

\subsubsection{Transcoding impairments}

In acquired aphasia and acalculia alike, lexical and morphosyntactic errors can often be observed when number words are presented auditorily or visually. 
These errors are sometimes accompanied by errors due to impaired verbal working memory. Number words are special because, unlike other long compound words (e.g., "football world championship"), semantics does not help to solve problems with the strict word order. In number words, all combinations of the elements of the number word dictionary are principally conceivable.

The two clinically most relevant transcoding pathways are between Arabic digits and spoken number words. When reading Arabic digits aloud, each of the three steps (identification of the digit chain, subsequent mental transformation into a sequence of words following fixed rules, utterance of the number word) can be disturbed.

In pure alexia, the initial (encoding) phase is impaired, while multi-digit numbers still can be compared with respect to their magnitude, because the non-dominant hemisphere can also encode Arabic digits and perform the magnitude comparison.

In lexical errors, a wrong element is selected from the same lexical number word class (ones 0-9; "teens" 11-19; decades 10-90), while the morphosyntactic structure (number word frame) of the target number word is preserved (e.g., 56 -> "seventy-six" or 411 -> "four hundred twelve"). In syntactical errors, an incorrect syntactic frame is generated which is filled with the "correct" number words corresponding to the digits in the Arabic numeral (e.g., 56 -> "five hundred and six").

When writing Arabic digits to dictation, not only lexical (e.g., "seventy-six" $\rightarrow$ 56) and syntactical errors occur (e.g., "five-hundred-and-six" -> 56), but also errors, in which the numerical word is transcoded in sections ("term-by-term") and the additive composition principle of multi-digit numbers is not correctly applied ("three-hundred-and-sixty-eight" -> “30068”).

Despite serious transcoding problems when verbalizing even single-digit numbers, a good understanding of the same numbers can be achieved by activating number-related semantic associations (e.g., 1945 -> "Hitler gone"). Patients with more severe naming disorders use the better-preserved up-counting technique for smaller numbers. Alternatively, fingers are used to show the number or the index finger is used to "imaginatively" write the Arabic numeral on a surface or in the air.

Acalculia and aphasia are often associated. Nevertheless, number processing problems and aphasia are not per se congruent (for an overview, see Willmes, 2008). Problems in reading and writing numbers occur mainly together, but also can be dissociated. Aphasic patients are typically better at choosing the larger number from a pair of Arabic multi-digit numbers than from a pair of spoken or written number words. 
Likewise, various transcoding errors are found in neuropsychological conditions different from aphasia. For instance, visual processing disorders like hemianopia or visual neglect can result in leaving out the leftmost digits (e.g., Hécaen et al., 1961) even without a similar problem in semantically adequate texts.

\subsubsection{Advantages and limitations of single-case studies}

One of the main limitations of single-case studies is that the data collected cannot necessarily be generalized to the broader population, so they might seem less meaningful. Criticism of generalizability, however, is of less relevance when the intention is one of theory discovery and model falsification as outlined by Shallice (1988) for the case of double associations or dissociations of cognitive processes. In this situation, qualitative knowledge of a specific case may be generalized to significant segments of the population (Kennedy, 1979). However, regarding statistical generalization, caveats are necessary. For instance, if statistical procedures initially designed for data from statistically independent replications are applied to data from a single subject (e.g., to differentiate reliably between classical, strong, or weak dissociations), assumptions of independence can become questionable (Willmes, 1990). Here, statistical and psychometric aspects have to be applied carefully, for example, by using additional normative data together with the binomial model for criterion-referenced measurement (Sergent, 1988).

In this vein, single-case designs can be used to apply, to build, and to a lesser extent, to test a theory as well as in the study of unique cases because they allow a more detailed data collection and can be conducted on rare cases where large samples of similar participants are not available.

\subsection{Developmental dyscalculia}

Developmental dyscalculia (DD) is an innate learning disability hampering the typical development of numerical and arithmetical competencies in children (Kaufmann \& Von Aster, 2012; Kucian \& von Aster, 2015) that can persist into adulthood. It results in a failure to achieve adequate proficiency in arithmetic despite normal intelligence, scholastic opportunity, emotional stability, and sufficient motivation (e.g., Shalev \& Gross-Tsur, 2001). Prevalence rates are rather high, ranging from 3.5\% to 6.5\% (Butterworth \& Kovas, 2013). With respect to the etiology of DD the core deficit hypothesis (suggesting a deficit in the core representation of number magnitude information) has long been the dominant view 
(e.g., Butterworth, 2005; Butterworth et al., 2011). Even though the heterogeneity of DD has long been observed empirically (e.g., Temple, 1991 for a differentiation of fact and procedural dyscalculia), the existence of subtypes of DD was acknowledged only recently in theoretical models on numerical development (e.g., Kucian \& Aster, 2015; Kaufmann \& Aster, 2012 as well as Kaufmann et al., 2013 for critical reviews).

At the brain level, the core deficit hypothesis implies that compared with typically developing children those with DD have deviant (intra)parietal fMRI responses (Ashkenazi et al., 2013; Kaufmann et al., 2011). Indeed, both overand underactivation of the IPS have been reported, which has been interpreted to reflect compensatory effortful functioning and deficient recruitment of number-relevant sites (i.e., deficient neuronal representation of numerosity), respectively. Furthermore, additional activation differences were found in (pre)frontal and occipital brain areas that were interpreted as reflecting domain-general compensatory mechanisms (Ashkenazi et al., 2013; Kaufmann et al., 2011; Peters \& De Smedt, 2018; see also McCaskey et al. (2018) for a longitudinal developmental fMRI study of number processing).

It is important to note that beyond the above-mentioned core deficit of number magnitude representation, math learning difficulties may also be caused by other dysfunctional systems. Alternative accounts are, for example, (i) deficient mapping processes between number symbols and their internal magnitude representations (Rousselle \& Noël, 2007; Rubinsten \& Henik, 2005), (ii) domain-general deficiencies such as attention (Ashkenazi \& Henik, 2010) and working memory (Rotzer et al., 2009; Toll et al., 2011), or (iii) a combined deficit of representing and manipulating numerical magnitude information (Ashkenazi et al., 2013). According to Ashkenazi et al. (2013), the latter hypothesis is especially apt to explain comorbid learning disorders (i.e., reading and math learning difficulties).

Fig. 2 provides a schematic overview of hypothetical subtypes of DD that is based on the TCM (Dehaene et al., 2003) and its postulate of three distinct types of (number) magnitude representations supported by distinct brain regions (i.e., numerical/analogue magnitude subtype: IPS, verbal subtype: left hemisphere language regions, spatial attentional subtype: posterior superior parietal lobe). According to this hypothetical model (Kaufmann \& Aster, 2012), the cognitive core deficiencies of these three subtypes of DD could not only be used to develop diagnostic marker tasks but could also provide an empirically validated framework to design efficient and tailored intervention tools. 


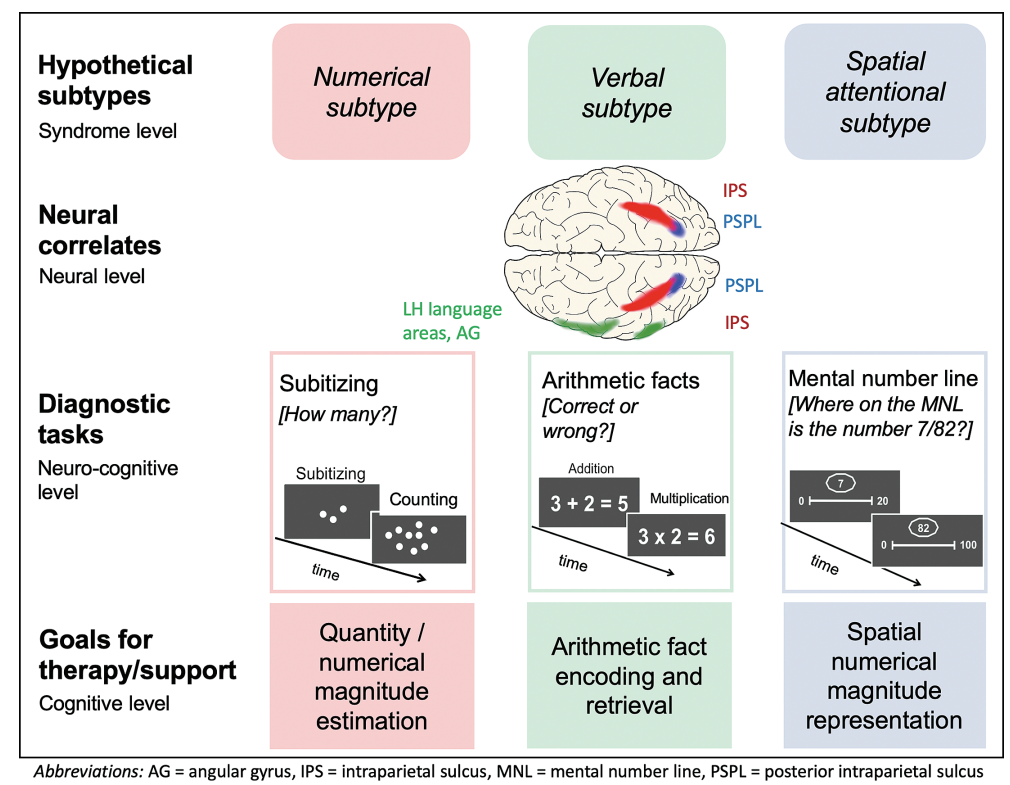

Fig. 2: Schematic representation of three distinct hypothetical subtypes of DD (differentiated at the behavioral and neural level, and assigned to specific diagnostic marker tasks and intervention goals).

\section{Language processing}

\subsection{Models for language processing}

Various cognitive components involved in language processing have been identified (e.g., language comprehension, reading and writing at the level of single words and in the context of sentences or functional communication) and described in various theoretical models (for language comprehension: e.g., the parallel interface model: Friederici, 2013, for reading: Coltheart, 1978; Caramazza et al., 1985; for writing: e.g., Ellis, 1982; 1988; Morton, 1980; for sentence production: Garrett, 1988; Levelt, 1993). These models have been elaborated on both typical and erroneous developmental trajectories of language processing (i.e., slip-of-the-tongue or slip-of-the-pen phenomena in the different language domains), and need to be differentiated from models describing impaired language processing in brain-injured patients (Ellis, 1982).

In principle, two types of models can be distinguished. First, in serial mod$e l s$, language processing is interpreted as a serial sequence of processing steps. 
The functional architecture of serial models is often graphically displayed by box-and-arrow graphs, in which a box represents a processing module, and an arrow represents the route in the direction of processing. Crucially, modules and routes are assumed to be affected and treated selectively (Ellis \& Young, 2013 for a review). Second, interactive models, also referred to as connectionist models, adopt hierarchically structured networks that can be activated in parallel (Dell, 1988; Dell et al., 1997; Dijkstra \& de Smedt, 1996). These networks are characterized by specific nodes and edges through which activations spread in all directions within the network.

Serial and interactive models are often interpreted as contradictory. Nevertheless, there are also language models that appear as hybrid (mixed) models (e.g., Levelt, 1993).

Recently, an association between behavior and neural correlates has been taken into account in models of language development in children. These models predominantly focused on theoretic descriptions of children's observable language behavior. Neural correlates played at best a very subordinate role and the description of the neural basis of language development still remains difficult (Friederici, 2011). However, based on neuroimaging findings, domain-general aspects underlying language processing have been identified as being already established in infants. In contrast, the domain-specific aspects emerge only gradually until they are fully established in young adulthood (Skeide \& Friederici, 2016).

\subsection{Neuropsychology}

In patients with acquired language disorders, speech or language is significantly impaired in one (or several) of the communication modalities (auditory comprehension, verbal expression, reading and writing, or functional communication).

\subsubsection{Acquired language disorders}

The generic term "acquired language disorders" covers various types of acquired functional impairments such as aphasia, alexia, and agraphia. The difficulties of people with aphasia can range from occasional trouble finding words to losing the ability to speak, read, or write while intelligence is unaffected. Alexia and agraphia can emerge as central and peripheral impairments of reading and writing. Central impairments often occur in association with other acquired language disorders such as aphasia (e.g., Delazer \& Bartha, 2001) and may affect reading and writing in all domains (i.e., letters and digits in the context of acalculia) and 
output modalities [i.e., oral spelling, handwriting, and typing (Beeson \& Rapcsak, 2015; Rapcsák, 2002)]. Peripheral impairments, in contrast, are related to the level of visual processing (alexia) or graphomotor planning and the execution of writing (Purcell et al., 2011).

The broad spectrum of symptoms as well as the combined and isolated occurrence of various speech and language deficits requires a precise understanding of typical language processes and their neural correlates in order to develop appropriate intervention approaches.

\subsubsection{Developmental dyslexia and dysgraphia}

Developmental dyslexia, like DD, belongs to the specific learning disabilities that develop in childhood and can persist into adulthood, and that cannot be explained by a child's chronological age, education, or IQ (APA, 2013). Both learning disabilities occur together more often than expected coincidentally (Wilson et al., 2015).

Developmental dyslexia describes the disability to sufficiently learn reading and/or writing despite an (above-) average intelligence and appropriate education. It is the most common subtype of learning disabilities with prevalence rates between 4 and 7\% in early childhood, depending on the criteria used (Landerl et al., 2009). Crucially, a gender discrepancy is noticeable with boys being more often affected than girls. Empirical data clearly indicate that dyslexia increases an individual's risk for school dropout, low educational achievement, and unemployment (Esser et al., 2002).

Children (and adults) with dyslexia have a deficit in the mechanism of phonological awareness, the ability to recognize, identify, and manipulate syllables and phonemes within spoken language (Shaywitz \& Shaywitz, 2012). They also struggle with handwriting aspects for various reasons, such as graphomotor planning and grapheme transcription (e.g., Kandel et al., 2017), correct spelling (e.g., Cidrim \& Madeiro, 2017, for a review), and writing fluency (e.g., Sumner et al., 2013; but see Martlewm, 1992). They also have difficulties in recognizing and correcting errors (e.g., Horowitz-Kraus \& Breznitz, 2011, for reading).

Etiological explanations for developmental dyslexia are twofold at least. On the one hand, difficulties due to basic deficits in (rapid) auditory (Tallal, 1980) and visual processing that arise from an impairment of the visual magnocellular system (Stein \& Walsh, 1997) are described. On the other hand, deficits in attentional (e.g., Facoetti et al., 2001) and automatization processes (e.g., Nicolson \& Fawcett, 2005) are discussed. Advocates of the latter theory argue that if cerebellar regions and the neural systems involved are affected (which are assumed 
to support automatization of basic articulatory and auditory skills), handwriting difficulties may arise (Nicolson \& Fawcett, 2011). Liberman (1973) and later Snowling (2000) related dyslexia to a deficit in phonological awareness. Although there is supporting empirical evidence for all of these theories, not all cognitive domains are simultaneously affected and several subtypes of developmental dyslexia are identified (Heim et al., 2008).

Brain imaging techniques identified dysfunctional neuronal networks in dyslexia mainly in left-hemispheric temporo-occipital, parieto-temporal and frontal brain regions (Ashkenazi et al., 2013). Both hypo- and hyperactivations have been reported (see Vandermosten et al., 2012 for a review), in terms of either reduced performance or compensatory strategies. Parts of this network are also associated with dyscalculia, for instance, the fusiform gyrus as well as the angular gyrus, which may explain comorbidity of both learning disorders (i.e., reading and math learning difficulties).

\section{Connectivity}

Both number and language processing are cases of distributed and connected processing in the human brain. As such, both arithmetic (e.g., Dehaene \& Cohen, 1997) and language processing (e.g., Hickok \& Poeppel, 2004) rely on widespread, separate, and overlapping networks in the human brain. This underscores the importance of white matter fiber connections between the specialized grey matter brain areas.

Analogous to the visual system with a dorsal "where" and a ventral "what" stream (Mishkin et al., 1983), it is assumed that language is processed in a dual system connecting Broca's and Wenicke's areas for language processing (Hickok \& Poeppel, 2004, 2007). The dorsal "where" stream along the arcuate fasciculus is dedicated to the mapping of auditory input to frontal articulatory networks (Hickok \& Poeppel, 2007), while the ventral "what" stream along the external/extreme capsule system is involved in language comprehension (Saura et al., 2008). The functional link between the arcuate fascicle and language dates back to Wernicke's (1874) suggestion that a lesion of association fibers connecting the sensory and motor speech areas leads to a disconnection syndrome characterized by a failure to repeat verbal information ("conduction aphasia"). This is in line with more recent models, which propose that the arcuate fascicle connects dorsally brain regions involved in sensorimotor processes supporting speech production and speech perception (Hickok \& Poeppel, 2007; Rauschecker \& Scott, 2009), that is, superior temporal gyrus with premotor cortex and posterior 
inferior frontal gyrus. In contrast, the ventral pathway connects Wernicke's area with Broca's area through the extreme and external capsule subserving meaning (Weiller et al., 2011).

Only in 2013, a first study investigated whether the fronto-parietal network from numerical cognition can be integrated into this framework of dorsal and ventral processing pathways (Klein et al., 2013). Using probabilistic fiber tracking, the authors showed that magnitude- and fact retrieval-related processing are indeed subserved by two largely separate networks, both of them following dorsal and ventral pathways. Nevertheless, even though distinct anatomically, these networks operate as a functionally integrated circuit for mental calculation. In 2016, the TCM was complemented by adding these neuro-structural connections between cortical areas related to magnitude processing and arithmetic fact retrieval (Klein et al., 2016). These amendments suggest that the general principles associated with dorsal ("doing") and ventral ("understand what you are doing”) processing streams, which seem to be instrumental for various domains (Rijntjes et al., 2012), can be adapted to number magnitude and fact retrieval processing in mental arithmetic.

So far, the work on the structural connectivity of these networks is primarily based on adults. Therefore, it is still not clear how any of these networks develop during childhood. Based on the work of Perani and colleagues (2011) for the case of language, the development of numerical competencies may most likely go hand-in-hand with brain maturation. In particular, studies from infancy to adulthood show later maturation of the dorsal compared to the ventral pathway (for an overview, see Friederici, 2012). While newborns have an adultlike ventral pathway at birth, the dorsal pathway that connects the temporal cortex to Broca's area develops later and is not fully matured at the age of seven (Perani et al., 2011). At this age, children still struggle with syntactically complex sentence processing. Therefore, it has been suggested that the degree of language competencies crucially depends on maturation of the dorsal pathway (Friederici, 2012). This would be in line with the idea children understand and process new numerical tasks first ventrally ("understanding what you are doing”), before more automated sequences of dorsal procedural processing can be established ("doing”).

Taken together, the two neuro-cognitive networks of magnitude representation and verbal representation are (1) functionally distinct and dissociable, (2) anatomically largely distinct, but they (3) nevertheless work together in a functionally integrated way. However, it is controversially discussed whether they (4) are exclusively processed dorsally (Amalric \& Dehaene, 2018) or follow general principles of dorsal and ventral processing (Klein et al., 2016). 


\section{Similarities in number and language processing}

The question of an association between aphasia and acalculia reflects an important topic in cognitive neuroscience: To what extents are higher cognitive functions such as numerical cognition based on language? The spectrum of assumptions ranges from the mediation of arithmetic by lexical and syntactic linguistic processes to the assumption that in adulthood, arithmetic can be independent of language. In the latest amendment to the TCM, Amalric and Dehaene (2018) argued that numerical and arithmetical processing are independent of "sentence-level language processing" and "general semantic thinking." In contrast to this view, Chomsky (2017; but see Corballis, 2017) argued that all thinking is verbal/linguistic thinking.

In particular, Amalric and Dehaene (2018) suggested that the behavioral dissociation between mathematical and linguistic skills is accompanied by a major neural dissociation between brain regions associated with math and regions involved in language processing and semantics. This idea would be in line with neuropsychological findings on differences between numbers and language (e.g., Jung et al., 2015). In a single-case study, Jung et al. (2015) observed impairments in writing letters while number writing was preserved. Single letters (e.g., "E") do not necessarily carry semantic information. Also, a random assembly of letters (e.g., "EHKU") does not necessarily produce a word that carries semantic meaning. In contrast, any number as well as any random assembly of numbers (e.g., "3," "3826") conveys meaning, namely, the numerical magnitude of the respective numbers. Hence, the authors suggested that semantic magnitude information of numbers can facilitate their processing. Importantly, in the therapy of the same patient, semantic cues were then added to each letter to support the retrieval of these letters. This additional semantic information indeed successfully facilitated letter writing.

In contrast, Chomsky (2017) argued that numerical knowledge is an adjunct of human linguistic ability. This view assumes a close link between the domains of language and number processing. Indeed, there are several theoretical accounts that emphasize the importance of language for the phylogenetic and ontogenetic development of the human faculty of number processing (e.g., Carey, 1998; and already, Henschen, 1919). For instance, Wiese (2007) concluded that "it is language that opened the way for numerical cognition," suggesting that "it is no accident that the same species that possesses the language faculty as a unique trait should also be the one that developed a systematic concept of number” (p. 758). Furthermore, both letters and numbers are linked to verbal linguistic 
information and both natural language and number processing share the need for a recursion operation that creates embedded tree structures (Hauser et al., 2002). There is also evidence that language and number processing share a number of crucial functional characteristics at the brain level such as an overlapping ventral network, at least for specific cognitive processes such as semantic classification (Willmes et al., 2014).

Probably, neither of the two proposals is sufficiently detailed to explain the numerical performance of both healthy and cognitively impaired participants. Rather, we suggest that it may be the type of the specific problem at hand and its difficulty that determines which system is used to solve the task or how we translate our thoughts into language. Very simple arithmetic problems may be retrieved verbally as facts from long-term memory or solved applying overlearned procedures (e.g., "rule of three"). More difficult arithmetic problems may be tackled with different strategies, which do not always have to be linguistic. Nevertheless, future studies are needed to provide evidence in favor of or against one of these accounts.

Taken together, there is an ongoing debate on the building blocks of number and language processing: on the one hand, there is the view that number processing and arithmetic are independent of language processing (Amalric \& Dehaene, 2018) and, on the other hand, there is the view that all thinking is linguistic thinking (Chomsky, 2017).

\section{References}

Amalric, Marie \& Dehaene, Stanislas (2018): Cortical circuits for mathematical knowledge: Evidence for a major subdivision within the brain's semantic networks. Philosophical Transactions of the Royal Society B: Biological Sciences 373, 1740. doi:10.1098/ rstb.2016.0515.

American Psychiatric Association. (2013). Diagnostic and statistical manual of mental disorders (DSM-5®). American Psychiatric Pub.

Ansari, Daniel \& Dhital, Bibek (2006): Age-related changes in the activation of the intraparietal sulcus during nonsymbolic magnitude processing: An event-related functional magnetic resonance imaging study. Journal of Cognitive Neuroscience. MIT Press 18 (11), 1820-1828.

Ardila, Alfredo \& Rosselli, Mónica (2002): Acalculia and dyscalculia. Neuropsychology Review 12 (4), 179-231. doi:10.1023/A:1021343508573.

Arsalidou, Marie, Pawliw-Levac, Matthew, Sadeghi, Mahsa \& Pascual-Leone, Juan (2018): Brain areas associated with numbers and calculations in children: Meta-analyses of $\mathrm{fMRI}$ studies. Developmental Cognitive Neuroscience 30, 239-250.

Arsalidou, Marie \& Taylor, Margot J. (2011): Is $2+2=4$ ? Meta-analyses of brain areas needed for numbers and calculations. Neurolmage 54 (3), 2382-2393. doi:10.1016/j. neuroimage.2010.10.009. http://dx.doi.org/10.1016/j.neuroimage.2010.10.009. 
Ashkenazi, Sarit, Black, Jessica M., Abrams, Daniel A., Hoeft, Fumiko \& Menon, Vinod (2013): Neurobiological underpinnings of math and reading learning disabilities. Journal of Learning Disabilities 46 (6), 549-569.

Ashkenazi, Sarit \& Henik, Avishai (2010): A disassociation between physical and mental number bisection in developmental dyscalculia. Neuropsychologia 48 (10), 2861-2868.

Basso, Anna, Caporali, Alessandra \& Faglioni, Pietro (2005): Spontaneous recovery from acalculia. Journal of the International Neuropsychological Society 11 (1), 99-107. doi:10.1017/S1355617705050113.

Beeson, Pélagie M. \& Rapcsak, Steven Z. (2015): Clinical diagnosis and treatment of spelling disorders. In The Handbook of Adult Language Disorders. Psychology Press, 133-154.

Bloechle, Johannes, Huber, Stefan, Bahnmueller, Julia, Rennig, Johannes, Willmes, Klaus, Cavdaroglu, Seda, Moeller, Korbinian \& Klein, Elise (2016): Fact learning in complex arithmetic - the role of the angular gyrus revisited. Human Brain Mapping 37 (9), 3061-3079. doi:10.1002/hbm.23226.

Bugden, Stephanie \& Ansari, Daniel (2011): Individual differences in children's mathematical competence are related to the intentional but not automatic processing of Arabic numerals. Cognition 118 (1), 32-44. doi:10.1016/j.cognition.2010.09.005. http://dx.doi. org/10.1016/j.cognition.2010.09.005.

Butterworth, Brian (2005): The development of arithmetical abilities. Journal of Child Psychology and Psychiatry 46 (1), 3-18.

Butterworth, Brian \& Kovas, Yulia (2013): Understanding neurocognitive developmental disorders can improve education for all. Science 340 (6130), 300-305.

Butterworth, Brian, Varma, Sashank \& Laurillard, Diana (2011): Dyscalculia: From brain to education. Science 332 (6033), 1049-1053. doi:10.1126/science.1201536.

Campbell, Jamie I.D. (1994): Architectures for numerical cognition. Cognition 53 (1), 1-44.

Cantlon, Jessica F., Brannon, Elizabeth M., Carter, Elizabeth J. \& Pelphrey, Kevin A. (2006): Functional imaging of numerical processing in adults and 4-y-old children. PLoS Biology 4 (5), 844-854. doi:10.1371/journal.pbio.0040125.

Caramazza, Alfonso, Miceli, Gabriele, Silveri, Maria Caterina \& Laudanna, Alessandro (1985): Reading mechanisms and the organisation of the lexicon: Evidence from acquired dyslexia. Cognitive Neuropsychology 2 (1), 81-114.

Carey, Susan (1998): Knowledge of number: Its evolution and ontogeny. Science 282 (5389), 641-642.

Chomsky, Noam (2017): Language architecture and its import for evolution. Neuroscience and Biobehavioral Reviews 81, 295-300. doi:10.1016/j.neubiorev.2017.01.053. https://doi. org/10.1016/j.neubiorev.2017.01.053.

Cidrim, Luciana \& Madeiro, Francisco (2017): Studies on spelling in the context of dyslexia: A literature review. Revista CEFAC. SciELO Brasil 19 (6), 842-854.

Cipolotti, Lisa \& Butterworth, Brian (1995): Toward a multiroute model of number processing: Impaired number transcoding with preserved calculation skills. Journal of Experimental Psychology: General 124 (4), 375-390. doi:10.1037/0096-3445.124.4.375.

Cipolotti, Lisa, Butterworth, Brian \& Denes, Gianfranco (1991): A specific deficit for numbers in a case of dense acalculia. Brain 114 (6), 2619-2637.

Cohen, Kadosh, Roi, Cohen Kadosh, Kathrin, Kaas, Amanda, Henik, Avishai \& Goebel, Rainer (2007): Notation-dependent and -independent representations of numbers in the parietal lobes. Neuron 53 (2), 307-314. doi:10.1016/j.neuron.2006.12.025. 
Coltheart, Max (1978): Lexical access in simple reading tasks. In Underwood, Geoffrey (ed.): Strategies of Information Processing. San Diego, CA: Academic Press, 151-216.

Corballis, Michael C. (2017): The evolution of language: Sharing our mental lives. Journal of Neurolinguistics 43, 120-132. doi:10.1016/j.jneuroling.2016.06.003. http://dx.doi.org/ 10.1016/j.jneuroling.2016.06.003.

Dehaene, Stanislas (1992): Varieties of numerical abilities. Cognition 44 (1-2), 1-42.

Dehaene, Stanislas \& Cohen, Laurent (1995): Towards an anatomical and functional model of number processing. Mathematical Cognition 1 (1), 83-120.

Dehaene, Stanislas \& Cohen, Laurent (1997): Cerebral pathways for calculation: Double dissociation between rote verbal and quantitative knowledge of arithmetic. Cortex 33 (2), 219-250. doi:10.1016/S0010-9452(08)70002-9.

Dehaene, Stanislas, Piazza, Manuela, Pinel, Philippe \& Cohen, Laurent (2003): Three parietal circuits for number processing. Cognitive Neuropsychology 20 (3-6), 487-506. doi:10.1080/02643290244000239.

Delazer, Margarete \& Bartha, Lisa (2001): Transcoding and calculation in aphasia. Aphasiology 15 (7), 649-679.

Delazer, Margarete \& Benke, Thomas (1997): Arithmetic facts without meaning. Cortex 33 (4), 697-710.

Delazer, Margarete, Domahs, Frank, Bartha, Lisa, Brenneis, Christian, Lochy, Aliette, Trieb, Thomas \& Benke, Thomas (2003): Learning complex arithmetic - An fMRI study. Cognitive Brain Research 18 (1), 76-88. doi:10.1016/j.cogbrainres.2003.09.005.

Delazer, Margarete, Karner, Elfriede, Zamarian, Laura, Donnemiller, Eveline \& Benke, Thomas (2006): Number processing in posterior cortical atrophy - A neuropsycholgical case study. Neuropsychologia 44 (1), 36-51. doi:10.1016/j.neuropsychologia.2005.04.013.

Delazer, Margarete, Zamarian, Laura, Benke, Thomas, Wagner, Michaela, Gizewski, Elke R. \& Scherfler, Christoph (2019): Is an intact hippocampus necessary for answering $3 \times 3$ ? Evidence from Alzheimer's disease. Brain and Cognition 134 (April), 1-8. doi:10.1016/j. bandc.2019.04.006.

Dell, Gary S. (1988): The retrieval of phonological forms in production: Tests of predictions from a connectionist model. Journal of Memory and Language 27 (2), 124-142.

Dell, Gary S., Schwartz, Myrna F., Martin, Nadine, Saffran, Eleanor M. \& Gagnon, Deborah A (1997): Lexical access in aphasic and nonaphasic speakers. Psychological Review 104 (4), 801-838. doi:10.1037/0033-295X.104.4.801.

Deloche, Gerard \& Willmes, Klaus (2000): Cognitive neuropsychological models of adult calculation and number processing: The role of the surface format of numbers. European Child and Adolescent Psychiatry 9 (2), 27-40. doi:10.1007/s007870070007.

Dijkstra, Ton \& Smedt, Koenraad de (1996): Computer models in psycholinguistics: An introduction. In Dijkstra, T., and de Smedt (eds.): Computational psycholinguistics. London: Taylor and Francis, 3-23.

Domahs, Frank \& Delazer, Margarete (2005): Concepts, procedures, and facts. Psychology 47 (1), 96-111. http://www.pabst-publishers.de/psychology-science/1-2005/ps_1_2005_ 96-111.pdf.

Eger, Evelyn, Sterzer, Philipp, Russ, Michael O., Giraud, Anne-Liese \& Kleinschmidt, Andreas (2003): A supramodal number representation in human intraparietal cortex. Neuron 37 (4), 719-726.

Ellis, Andrew W. (1982): Spelling and writing (and reading and speaking). In Ellis, Andrew W. (ed.): Normality and Pathology in Cognitive Functions. London: Academic Press, 113-146. 
Ellis, Andrew W. (1988): Normal writing processes and peripheral acquired dysgraphias. Language and Cognitive Processes 3 (2), 99-127.

Ellis, A. W., \& Young, A. W. (2013): Human cognitive neuropsychology: A textbook with readings. Hove, East Sussex: Psychology Press

Esser, Günter, Wyschkon, Anne \& Schmidt, Martin H. (2002): Was wird aus Achtjährigen mit einer Lese-und Rechtschreibstörung. Zeitschrift für Klinische Psychologie und Psychotherapie; Forschung und Praxis 31 (4), 235-242.

Facoetti, Andrea, Turatto, Massimo, Lorusso, Maria Luisa \& Mascetti, Gian Gastone (2001): Orienting of visual attention in dyslexia: Evidence for asymmetric hemispheric control of attention. Experimental Brain Research 138 (1), 46-53.

Friederici, Angela D. (2011): The brain basis of language processing: From structure to function. Physiological Reviews 91 (4), 1357-1392. doi:10.1152/physrev.00006.2011.

Friederici, Angela D. (2012): Language development and the ontogeny of the dorsal pathway. Frontiers in Evolutionary Neuroscience 4 (FEB), 1-7. doi:10.3389/fnevo.2012.00003.

Friederici, Angela D. (2013): Kognitive Strukturen des Sprachverstehens. Berlin, Heidelberg: Springer.

Garrett, Merrill F. (1988). Processes in language production. In F. Newmeyer (Ed.), Linguistics: The Cambridge Survey (pp. 69-96). Cambridge: Cambridge University Press. doi:10.1017/ CB09780511621062.004

Geary, David C. (1993): Mathematical disabilities: Cognitive, neuropsychological, and genetic components. Psychological Bulletin. doi:10.1037/0033-2909.114.2.345.

Hauser, Marc D., Chomsky, Noam \& Tecumseh Fitch, W. (2002): The faculty of language: What is it, who has it, and how did it evolve?. Science 298 (5598), 1569-1579.

Hécaen, Henri, Angelergues, René \& Houillier, S. (1961): The clinical varieties of acalculias during retrorolandic lesions: Statistical approach to the problem. Revue Neurologique 105, 85-103.

Heim, Stefan, Tschierse, Julia, Amunts, Katrin, Wilms, Marcus, Vossel, Simone, Willmes, Klaus, Grabowska, Anna \& Huber, Walter (2008): Cognitive subtypes of dyslexia. Acta Neurobiologiae Experimentalis 68 (1), 73-82.

Henschen, Salomon Eberhard (1919): Über Sprach-, Musik- und Rechenmechanismen und ihre Lokalisationen im Großhirn. Zeitschrift für die gesamte Neurologie und Psychiatrie 52 (1), 273-298. doi:10.1007/BF02872428.

Hickok, Gregory \& Poeppel, David (2004): Dorsal and ventral streams: A framework for understanding aspects of the functional anatomy of language. Cognition 92 (1-2), 67-99.

Hickok, Gregory \& Poeppel, David (2007): The cortical organization of speech processing. Nature Reviews Neuroscience 8 (5), 393-402. doi:10.1038/nrn2113.

Horowitz-Kraus, Tzipi \& Breznitz, Zvia (2011): Error Detection Mechanism for Words and Sentences: A comparison between readers with dyslexia and skilled readers. International Journal of Disability, Development and Education 58 (1), 33-45.

Jung, Stefanie, Halm, Katja, Huber, Walter, Willmes, Klaus \& Klein, Elise (2015): What letters can "learn" from Arabic digits - fMRI-controlled single case therapy study of peripheral agraphia. Brain and Language 149 (July 2015), 13-26. doi:10.1016/j.bandl.2015.06.003.

Kandel, Sonia, Lassus-Sangosse, Delphine, Grosjacques, Géraldine \& Perret, Cyril (2017): The impact of developmental dyslexia and dysgraphia on movement production during word writing. Cognitive Neuropsychology 34 (3-4), 219-251.

Kaufmann, Liane \& Aster, Michael von (2012): Diagnostik und Intervention bei Rechenstörung. Deutsches Ärzteblatt International 109 (45), 767-778. doi:10.3238/arztebl.2012.0767. 
Kaufmann, Liane, Koppelstaetter, Florian, Siedentopf, Christian, Haala, Ilka, Haberlandt, Edda, Zimmerhackl, Lothar-Bernd, Felber, Stefan \& Ischebeck, Anja (2006): Neural correlates of the number-size interference task in children. Neuroreport. Europe PMC Funders 17 (6), 587.

Kaufmann, Liane, Mazzocco, Michèle M., Dowker, Ann, Aster, Michael von, Göbel, Silke M., Grabner, Roland H., Henik, Avishai et al. (2013): Dyscalculia from a developmental and differential perspective. Frontiers in Psychology 4 (Aug), 1-5. doi:10.3389/fpsyg.2013.00516.

Kaufmann, Liane, Wood, Guilherme, Rubinsten, Orly \& Henik, Avishai (2011): Meta-analyses of developmental fMRI studies investigating typical and atypical trajectories of number processing and calculation. Developmental Neuropsychology 36 (6), 763-787. doi:10.1080/87565641.2010.549884.

Kennedy, Mary M. (1979): Generalizing from single case studies. Evaluation Quarterly 3 (4), 661-678. doi:https://doi.org/10.1177/0193841X7900300409.

Klein, Elise, Korbinian, Moeller, Nuerk, Hans Christoph \& Willmes, Klaus (2010): On the neurocognitive foundations of basic auditory number processing: An fMRI study. Behavioral and Brain Functions 6 (1), 42.

Klein, Elise, Moeller, Korbinian, Glauche, Volkmar, Weiller, Cornelius \& Willmes, Klaus (2013): Processing pathways in mental arithmetic-evidence from probabilistic fiber tracking. PLOS ONE 8, 1. doi:10.1371/journal.pone.0055455.

Klein, Elise, Moeller, Korbinian, Huber, Stefan, Willmes, Klaus, Kiechl-Kohlendorfer, Ursula \& Kaufmann, Liane (2018): Gestational age modulates neural correlates of intentional, but not automatic number magnitude processing in children born preterm. International Journal of Developmental Neuroscience 65 (October 2017), 38-44. doi:10.1016/j. ijdevneu.2017.10.004. http://dx.doi.org/10.1016/j.ijdevneu.2017.10.004.

Klein, Elise, Suchan, Julia, Moeller, Korbinian, Karnath, Hans Otto, Knops, André, Wood, Guilherme, Nuerk, Hans Christoph \& Willmes, Klaus (2016): Considering structural connectivity in the triple code model of numerical cognition: Differential connectivity for magnitude processing and arithmetic facts. Brain Structure and Function 221 (2), 979-995. doi:10.1007/s00429-014-0951-1.

Klein, Elise, Willmes, Klaus, Bieck, Silke M., Bloechle, Johannes \& Moeller, Korbinian (2019): White matter neuro-plasticity in mental arithmetic: Changes in hippocampal connectivity following arithmetic drill training. Cortex 114, 115-123. doi:10.1016/j.cortex.2018.05.017. https://doi.org/10.1016/j.cortex.2018.05.017.

Kosc, Ladislav (1974): Developmental dyscalculia. Journal of Learning Disabilities 7 (3), 164-177. Kucian, Karin \& Aster, Michael von (2015): Developmental dyscalculia. European Journal of Pediatrics. Springer 174 (1), 1-13.

Kucian, Karin \& Kaufmann, Liane (2009): A developmental model of number representation. Behavioral and Brain Sciences. Cambridge University Press 32 (3-4), 340-341.

Landerl, Karin, Fussenegger, Barbara, Moll, Kristina \& Willburger, Edith (2009): Dyslexia and dyscalculia: Two learning disorders with different cognitive profiles. Journal of Experimental Child Psychology 103 (3), 309-324. doi:10.1016/j.jecp.2009.03.006.

LeFevre, Jo-Anne, Bisanz, Jeffrey, Daley, Karen E, Buffone, Lisa, Greenham, Stephanie L \& Sadesky, Gregory S (1996): Multiple routes to solution of single-digit multiplication problems. Journal of Experimental Psychology: General. American Psychological Association 125 (3), 284.

Lemer, Cathy, Dehaene, Stanislas, Spelke, Elizabeth \& Cohen, Laurent (2003): Approximate quantities and exact number words: Dissociable systems. Neuropsychologia 41 (14), 1942-1958. doi:10.1016/S0028-3932(03)00123-4. 
Levelt, Willem J M. (1993): Speaking: From intention to articulation. 1, Cambridge(MA): MIT press. Liberman, Isabelle Y. (1973): Segmentation of the spoken word and reading acquisition. In Bulletin of the Maryland: Orton Society, Inc. JSTOR, 65-77.

Martlewm, Margaret (1992): Handwriting and spelling: Dyslexic children's abilities compared with children of the same chronological age and younger children of the same spelling level. British Journal of Educational Psychology. Wiley Online Library 62 (3), 375-390.

McCaskey, Ursina, von Aster, Michael, Maurer, Urs, Martin, Ernst, Ruth O’Gorman, Tuura \& Karin, Kucian (2018): Longitudinal brain development of numerical skills in typically developing children and children with developmental dyscalculia. Frontiers in Human Neuroscience. Frontiers 11, 629.

McCloskey, Michael (1992): Cognitive mechanisms in numerical processing: Evidence from acquired dyscalculia. Cognition 44 (1-2), 107-157.

McCloskey, Michael, Caramazza, Alfonso \& Basili, Annamaria (1985): Cognitive mechanisms in number processing and calculation: Evidence from dyscalculia. Brain and cognition. Elsevier 4 (2), 171-196.

Menon, Vinod (2016): Memory and Cognitive Control Circuits in Mathematical Cognition and Learning. Progress in Brain Research. 1st edn. 227, Elsevier B.V., doi:10.1016/bs. pbr.2016.04.026. http://dx.doi.org/10.1016/bs.pbr.2016.04.026.

Mishkin, Mortimer, Ungerleider, Leslie G. \& Macko, Kathleen A. (1983): Object vision and spatial vision: Two cortical pathways. Trends in Neurosciences 6 (C), 414-417. doi:10.1016/0166-2236(83)90190-X.

Morton, John (1980): The logogen model and orthographic structure. In Frith, U. (Ed.): Cognitive Approaches in Spelling. London: Academic Press, 117-134.

Naccache, Lionel \& Dehaene, Stanislas (2001): Unconscious semantic priming extends to novel unseen stimuli. Cognition 80 (3), 215-229. doi:10.1016/S0010-0277(00)00139-6.

Nicolson, Roderick I \& Fawcett, Angela J (2005): Developmental dyslexia, learning and the cerebellum. In Neurodevelopmental Disorders. Springer, 19-36.

Nicolson, Roderick I \& Fawcett, Angela J (2011): Dyslexia, dysgraphia, procedural learning and the cerebellum. In Cortex: A Journal Devoted to the Study of the Nervous System and Behavior. Elsevier Masson SAS.

Perani, Daniela, Saccuman, Maria C, Scifo, Paola, Anwander, Alfred, Spada, Danilo, Baldoli, Cristina, Poloniato, Antonella, Lohmann, Gabriele \& Friederici, Angela D (2011): Neural language networks at birth. Proceedings of the National Academy of Sciences. National Acad Sciences 108 (38), 16056-16061.

Pesenti, Mauro, Depoorter, Nathalie \& Seron, Xavier (2000): Noncommutability of the N+ 0 arithmetical rule: A case study of dissociated impairment. Cortex. Elsevier 36 (3), 445-454.

Peters, Lien \& Bert, De Smedt (2018): Arithmetic in the developing brain: A review of brain imaging studies. Developmental Cognitive Neuroscience. Elsevier 30, 265-279.

Peters, Lien, Polspoel, Brecht, Op de Beeck, Hans \& De Smedt, Bert (2016): Brain activity during arithmetic in symbolic and non-symbolic formats in 9-12 year old children. Neuropsychologia. Elsevier 86, 19-28.

Piazza, Manuela, Pinel, Philippe, Le Bihan, Denis \& Dehaene, Stanislas (2007): A magnitude code common to numerosities and number symbols in human intraparietal cortex. Neuron 53 (2), 293-305. doi:10.1016/j.neuron.2006.11.022.

Purcell, Jeremy, Turkeltaub, Peter E, Eden, Guinevere F \& Rapp, Brenda (2011): Examining the central and peripheral processes of written word production through meta-analysis.

Frontiers in Psychology. Frontiers 2, 239. 
Rapcsák, Tamás (2002): On minimization on stiefel manifolds. European Journal of Operational Research. Elsevier 143 (2), 365-376.

Ratinckx, Elie, Nuerk, Hans Christoph, van Dijck, Jean Philippe \& Willmes, Klaus (2006): Effects of interhemispheric communication on two-digit arabic number processing. Cortex 42 (8), 1128-1137. doi:10.1016/S0010-9452(08)70225-9.

Rauschecker, Josef P. \& Scott, Sophie K. (2009): Maps and streams in the auditory cortex: Nonhuman primates illuminate human speech processing. Nature Neuroscience 12 (6), 718-724. doi:10.1038/nn.2331.

Rijntjes, Michel, Weiller, Cornelius, Bormann, Tobias \& Musso, Mariacristina (2012): The dual loop model: Its relation to language and other modalities. Frontiers in Evolutionary Neuroscience 4 (JUL). doi:10.3389/fnevo.2012.00009.

Rivera, S. M., Reiss, A. L., Eckert, M. A. \& Menon, V. (2005): Developmental changes in mental arithmetic: Evidence for increased functional specialization in the left inferior parietal cortex. Cerebral Cortex 15 (11), 1779-1790. doi:10.1093/cercor/bhi055.

Rosenberg-Lee, Miriam, luculano, Teresa, Bae, Se Ri, Richardson, Jennifer, Qin, Shaozheng, Jolles, Dietsje \& Menon, Vinod (2018): Short-term cognitive training recapitulates hippocampal functional changes associated with one year of longitudinal skill development. Trends in Neuroscience and Education. Elsevier GmbH 10 (June 2017). 19-29. doi:10.1016/j.tine.2017.12.001. https://doi.org/10.1016/j.tine.2017.12.001.

Rotzer, Stephanie, Loenneker, Thomas, Kucian, Karin, Martin, Ernst, Klaver, Peter \& Michael, von Aster (2009): Dysfunctional neural network of spatial working memory contributes to developmental dyscalculia. Neuropsychologia 47 (13), 2859-2865. doi:10.1016/j. neuropsychologia.2009.06.009.

Rousselle, Laurence \& Marie-Pascale, Noël (2007): Basic numerical skills in children with mathematics learning disabilities: A comparison of symbolic vs non-symbolic number magnitude processing. Cognition. Elsevier 102 (3), 361-395.

Rubinsten, Orly \& Henik, Avishai (2005): Automatic activation of internal magnitudes: A study of developmental dyscalculia. Neuropsychology. American Psychological Association 19 (5), 641.

Saura, Dorothee, Kreher, Björn W., Schnell, Susanne, Kümmerera, Dorothee, Kellmeyera, Philipp, Vrya, Magnus Sebastian, Umarova, Roza et al. (2008): Ventral and dorsal pathways for language. Proceedings of the National Academy of Sciences of the United States of America 105 (46), 18035-18040, doi:10.1073/pnas.0805234105.

Sergent, Justine (1988): Some theoretical and methodological issues in neuropsychologial research. In Boller, F., Grafman, J. (eds): Handbook of Neuropsychology. Amsterdam: Elsevier.

Shalev, Ruth S \& Gross-Tsur, Varda (2001): Developmental dyscalculia. Pediatric Neurology. Elsevier 24 (5), 337-342.

Shallice, Tim. (1988): From Neuropsychology to Mental Structure. Cambridge: Cambridge University Press.

Shaywitz, Sally E \& Shaywitz, Bennett A (2012): Dyslexia and reading disorders. In Psychopathology of Childhood and Adolescence: A Neuropsychological Approach. Springer Publishing Company, 127.

Skeide, Michael A \& Friederici, Angela D (2016): The ontogeny of the cortical language network. Nature Reviews Neuroscience. Nature Publishing Group 17 (5), 323.

Snowling, Margaret J. (2000): Dyslexia. Oxford: Blackwell publishing.

Stein, John \& Walsh, Vincent (1997): To see but not to read; the magnocellular theory of dyslexia. Trends in Neurosciences. Elsevier 20 (4), 147-152. 
Sumner, Emma, Connelly, Vincent \& Barnett, Anna L (2013): Children with dyslexia are slow writers because they pause more often and not because they are slow at handwriting execution. Reading and Writing. Springer 26 (6), 991-1008.

Tallal, Paula (1980): Auditory temporal perception, phonics, and reading disabilities in children. Brain and Language. Elsevier 9 (2), 182-198.

Temple, Christine M. (1991): Procedural dyscalculia and number fact dyscalculia: Double dissociation in developmental dyscalculia. Cognitive Neuropsychology. Taylor \& Francis 8 (2), 155-176.

Toll, Sylke W M, Van der Ven, Sanne H G, Kroesbergen, Evelyn H \& Van Luit, Johannes E H (2011): Executive functions as predictors of math learning disabilities. Journal of Learning Disabilities. Sage Publications Sage CA: Los Angeles, CA 44 (6), 521-532.

Vandermosten, Maaike, Boets, Bart, Wouters, Jan \& Pol, Ghesquière (2012): A qualitative and quantitative review of diffusion tensor imaging studies in reading and dyslexia. Neuroscience and Biobehavioral Reviews. Elsevier 36 (6), 1532-1552.

Warrington, Elizabeth K. (1982): The fractionation of arithmetical skills: A single case study. The Quarterly Journal of Experimental Psychology Section A. SAGE Publications Sage UK: London, England 34 (1), 31-51.

Weiller, Cornelius, Bormann, Tobias, Saur, Dorothee, Musso, Mariachristina \& Rijntjes, Michel (2011): How the ventral pathway got lost - And what its recovery might mean. Brain and Language 118 (1-2), 29-39. doi:10.1016/j.bandl.2011.01.005.

Wernicke, Carl (1874): Der aphasische Symptomencomplex: Eine psychologische Studie auf anatomischer Basis. Breslau: Max Cohn \& Weigert.

Wiese, Heike (2007): The co-evolution of number concepts and counting words. Lingua 117 (5), 758-772. doi:10.1016/j.lingua.2006.03.001.

Willmes, Klaus (1990): Statistical methods for a single-case study approach to aphasia therapy research. Aphasiology 4 (4), 415-436.

Willmes, Klaus (2008): Acalculia. In: Goldenberg, G, Miller BL (Hrsg). Handbook of clinical neurology, Bd 88 (3rd series). Neuropsychology and behavioral neurology. Elsevier: London. 339-358.

Willmes, Klaus, Moeller, Korbinian \& Klein, Elise (2014): Where numbers meet words: A common ventral network for semantic classification. Scandinavian Journal of Psychology 55 (3), 202-211. doi:10.1111/sjop.12098.

Wilson, Anna J, Andrewes, Stuart G, Struthers, Helena, Rowe, Victoria M, Bogdanovic, Rajna \& Waldie, Karen E (2015): Dyscalculia and dyslexia in adults: Cognitive bases of comorbidity. Learning and Individual Differences. Elsevier 37, 118-132.

Zaunmuller, Luisa, Domahs, Frank, Dressel, Katharina, Lonnemann, Jan, Klein, Elise, Ischebeck, Anja \& Willmes, Klaus (2009): Rehabilitation of arithmetic fact retrieval via extensive practice: A combined fMRI and behavioural case-study. Neuropsychological Rehabilitation 19 (3), 422-443. doi:10.1080/09602010802296378. 